\title{
On-line list colouring of graphs
}

\author{
Xuding Zhu* \\ Department of Applied Mathematics \\ National Sun Yat-sen University \\ Kaohsiung, Taiwan 80424 \\ and \\ National Center for Theoretical Sciences, Taiwan \\ zhu@math.nsysu.edu.tw \\ Submitted: May 27, 2009; Accepted: Oct 7, 2009; Published: Oct 17, 2009 \\ Mathematics Subject Classification: 05C15
}

\begin{abstract}
This paper studies on-line list colouring of graphs. It is proved that the online choice number of a graph $G$ on $n$ vertices is at most $\chi(G) \ln n+1$, and the on-line $b$-choice number of $G$ is at most $\frac{e \chi(G)-1}{e-1}(b-1+\ln n)+b$. Suppose $G$ is a graph with a given $\chi(G)$-colouring of $G$. Then for any $(\chi(G) \ln n+1)$-assignment $L$ of $G$, we give a polynomial time algorithm which constructs an $L$-colouring of $G$. For any $\left(\frac{e \chi(G)-1}{e-1}(b-1+\ln n)+b\right)$-assignment $L$ of $G$, we give a polynomial time algorithm which constructs an $(L, b)$-colouring of $G$. We then characterize all on-line 2-choosable graphs. It is also proved that a complete bipartite graph of the form $K_{3, q}$ is on-line 3-choosable if and only if it is 3-choosable, but there are graphs of the form $K_{6, q}$ which are 3-choosable but not on-line 3-choosable. Some open questions concerning on-line list colouring are posed in the last section.
\end{abstract}

\section{Introduction}

Suppose $G$ is a graph, $f$ and $g$ are two functions from $V(G)$ to $\mathbb{N}$ (we use the convention that $\mathbb{N}=\{0,1,2, \ldots\})$, with $f(v) \geqslant g(v)$ for all $v \in V(G)$. An $f$-assignment of $G$ is a mapping $L$ which assigns to each vertex $v$ of $G$ a set $L(v)$ of $f(v)$ positive integers as permissible colours. A $g$-colouring of $G$ is a mapping $S$ which assigns to each vertex $v$ of $G$ a set $S(v)$ of $g(v)$ colours such that for any two adjacent vertices $u$ and $v, S(v) \cap S(u)=\emptyset$. Given a list assignment $L$ of $G$, an $(L, g)$-colouring of $G$ is a $g$-colouring $S$ of $G$ such

\footnotetext{
${ }^{*}$ This research was partially supported by the National Science Council under grant NSC97-2115-M-
} 110-008-MY3 
that for each vertex $v, S(v) \subseteq L(v)$. We say $G$ is $(L, g)$-colourable if there exists an $(L, g)$-colouring of $G$. We say $G$ is $(f, g)$-choosable if for every $f$-assignment $L, G$ is $(L, g)$-colourable. Some special cases of $(f, g)$-choosability have their own names:

- If $g \equiv b$ is a constant function, then $(f, g)$-choosable is called $(f, b)$-choosable.

- If $f \equiv m$, then $(f, b)$-choosable is called $(m, b)$-choosable.

- If $b=1$, then $(f, b)$-choosable is called $f$-choosable.

- If $f \equiv m$, then $f$-choosable is called $m$-choosable.

The choice number $\operatorname{ch}(G)$ of $G$ is the minimum $m$ for which $G$ is $m$-choosable. The $b$-choice number $\operatorname{ch}_{b}(G)$ of $G$ is the minimum $m$ for which $G$ is $(m, b)$-choosable. List colouring of graphs was introduced in the 1970s by Vizing [17] and independently by Erdös, Rubin and Taylor [6]. The subject offers a large number of challenging problems and has attracted an increasing attention since 1990. Readers are referred to [16] for a comprehensive survey on results and open problems. In this paper, we consider a variation of the list colouring problem: on-line list colouring of graphs.

List assignments and colourings of $G$ can be defined alternately as follows: A list assignment $L$ of a graph $G$ can be given as a sequence $\left(V_{1}, V_{2}, \ldots, V_{m}\right)$ of subsets of $V(G)$, where $V_{i}=\{v: i \in L(v)\}$. In other words, $v \in V_{i}$ if $i$ is a permissible colour of $v$. In the following, we write a list assignment $L$ as $L=\left(V_{1}, V_{2}, \ldots, V_{m}\right)$. Given a list assignment $L=\left(V_{1}, V_{2}, \ldots, V_{m}\right)$, an $L$-colouring of $G$ is equivalent to a sequence $\left(X_{1}, X_{2}, \ldots, X_{m}\right)$ such that each $X_{i}$ is an independent set contained in $V_{i}$ (it is allowed that $X_{i}$ be empty). If $v \in X_{i}$, then we say $v$ is coloured with colour $i$.

This alternate definition motivates the definition of the on-line list colouring of graphs, which is defined through a two-person game.

Definition 1 Given a graph $G$ and two mappings $f, g: V(G) \rightarrow \mathbb{N}$, the on-line $(f, g)$ list colouring game on $G$ is a game with two players: Thief and Police. In the ith step, Thief chooses a non-empty subset $V_{i}$ of $V(G)$, and Police chooses an independent set $X_{i}$ contained in $V_{i}$. A vertex $v$ is finished if $v$ is contained in $f(v)$ of the $V_{j}$ 's. When Thief chooses the set $V_{i}$, it is required that $V_{i}$ contains only non-finished vertices. If for some integer $m$, at the end of the mth step, there is a finished vertex $v$ which is contained in less than $g(v)$ of $X_{j}$ 's (for $j=1,2, \ldots, m$ ), then Thief wins the game. Otherwise, at some step, each vertex $v$ becomes finished and is contained in $g(v)$ of the $X_{i}$ 's. In this case, Police wins the game.

Thus in the game, Thief is required to give $f(v)$ permissible colours to vertex $v$ and Police needs to colour $v$ with $g(v)$ permissible colours, under the restriction that no colour is assigned to two adjacent vertices. Police wins the game if every vertex $v$ is successfully coloured with $g(v)$ colours.

Here is a more entertaining explanation of the $(f, g)$-list colouring game: Each vertex of $G$ is a thief. Each thief $v$ is planned to commit theft $f(v)$ times. Police is watching 
these thieves. If a thief commit a theft, Police can catch him. However, if two thieves commit theft at the same time, and these two thieves are joined by an edge in the graph, then Police can catch at most one of them. If thief $v$ is caught $g(v)$ times, then he will be put into prison. At each round, Thief decides which thieves go out to commit theft, and Police, after knowing which thieves are out, decides which thieves to be caught. The goal of Police is to put all the thieves into prison. If he achieves this goal, then he is the winner. Otherwise, Thief is the winner.

Definition 2 Suppose $f, g: V(G) \rightarrow \mathbb{N}$. We say $G$ is on-line $(f, g)$-choosable if Police has a winning strategy in the $(f, g)$-list colouring game on $G$.

For positive integers $m, b, G$ is on-line $(f, b)$-choosable means that $G$ is on-line $(f, g)$ choosable for the constant function $g \equiv b$; $G$ is on-line $f$-choosable means that $G$ is on-line $(f, 1)$-choosable; $G$ is on-line $m$-choosable means that $G$ on-line $f$-choosable for the constant function $f \equiv m ; G$ is on-line $(m, b)$-choosable means that $G$ is on-line $(f, b)$ choosable for the constant function $f \equiv m$.

The on-line choice number $\mathrm{ch}^{\mathrm{OL}}(G)$ of $G$ is the minimum $m$ for which $G$ is on-line $m$-choosable. The on-line b-choice number $\operatorname{ch}_{b}^{\mathrm{OL}}(G)$ of $G$ is the minimum $m$ for which $G$ is on-line $(m, b)$-choosable.

List colouring of graphs has been studied extensively in the literature. The on-line list colouring of graphs is recently studied in [11] under a different name: the Paint and Correct game. It follows from the definition that for any graph $G, \operatorname{ch}^{\mathrm{OL}}(G) \geqslant \operatorname{ch}(G)$ and $\operatorname{ch}_{b}^{\mathrm{OL}}(G) \geqslant \operatorname{ch}_{b}(G)$. However, it is shown in $[8,12,13,11]$ that many upper bounds for the choice number of a graph remain upper bounds for its on-line choice number. For example, the on-line choice number of planar graphs is at most 5 , the on-line choice number of the line graph $L(G)$ of a bipartite graph $G$ is $\Delta(G)$, and if $G$ has an orientation in which the number of even eulerian subgraphs differs from the number of odd eulerian subgraphs and $f(x)=d^{+}(x)+1$, then $G$ is on-line $f$-choosable.

The relation between the choice number, the $b$-choice number and the chromatic number of a graph has been studied in the literature. In [1], Alon proved that for any positive integers $m$ and $r \geqslant 2$, the complete $r$-partite graph $K_{m \star r}$ (with each partite set of cardinality $m$ ) satisfies

$$
c_{1} r \ln m \leqslant c h\left(K_{m \star r}\right) \leqslant c_{2} r \ln m
$$

where $c_{2}$ is a sufficiently large constant and $c_{1}$ is a sufficiently small positive constant. The upper bound remains true for any complete $r$-partite graph $G$, and hence for any graph $G$ on $n$ vertices,

$$
\operatorname{ch}(G) \leqslant c_{2} \chi(G) \ln n .
$$

The constant $c_{2}$ is given explicitly in [7] as 948 . For the $b$-choice number, it is proved in [7] that for a graph $G$ on $n$ vertices,

$$
c h_{b}(G) \leqslant 948 \chi(G)\left(b+\ln \left(\frac{n}{\chi(G)}+1\right)\right) .
$$

The proofs of the results in $[1,7]$ use the probabilistic method. No explicit algorithm is given to construct an $L$-colouring for a given list assignment $L$. 
In this paper, we are interested in the relation between the on-line choice number, the on-line $b$-choice number and the chromatic number of a graph. We prove that if $G$ is a graph on $n$ vertices, then

$$
\operatorname{ch}(G) \leqslant \operatorname{ch}^{\mathrm{OL}}(G) \leqslant \chi(G) \ln n+1 .
$$

For on-line $b$-list colouring of graphs, we prove that

$$
c h_{b}(G) \leqslant \operatorname{ch}_{b}^{\mathrm{OL}}(G) \leqslant \frac{e \chi(G)-1}{e-1}(b-1+\ln n)+b .
$$

Here $e$ is the base of the natural logarithm. The proofs for the upper bounds are conceptually simpler, and give an algorithm, with running time $O(n \ln n)$, that constructs, for any $(\chi(G) \ln n+1)$-assignment $L$ of $G$, an $L$-colouring of $G$, and for any $\left(\frac{e}{e-1} \chi(G)(b-1+\ln n)+b\right)$-assignment $L$ of $G$, an $(L, b)$-colouring of $G$.

The family of 2-choosable graphs was characterized by Erdös, Rubin and Taylor in [6]. We shall characterize all graphs $G$ with $\operatorname{ch}^{\mathrm{OL}}(G) \leqslant 2$. It turns out that not all 2-choosable graphs are on-line 2-choosable.

We also study on-line 3-choosability of complete bipartite graphs. It is proved that a complete bipartite graph of the form $K_{3, q}$ is on-line 3-choosable if and only if it is 3choosable, but there are graphs of the form $K_{6, q}$ which are 3-choosable but not on-line 3-choosable.

For a subset $U$ of $V(G)$, let $f|U, g| U$ be the restrictions of $f, g$ to $U$. We say $G[U]$ is on-line $(f, g)$-choosable if $G[U]$ is on-line $(f|U, g| U)$-choosable. Note that if $g(x)=0$, then no colour needs to be assigned to $x$ and we may ignore this vertex and simply consider the graph $G-x$. However, for the sake of notation, it is more convenient to keep the vertex there.

\section{Basic properties}

Given a subset $X$ of $V$, let $\delta_{X}$ be the characteristic function of $X$, i.e., $\delta_{X}(x)=1$ if $x \in X$ and $\delta_{X}(x)=0$ otherwise. If $U=\{x\}$ we write $\delta_{x}$ instead of $\delta_{\{x\}}$. In the following, we frequently difference of two functions, and multiplication of a function by a constant. For example, for a mapping $f: V(G) \rightarrow \mathbb{N}, h=f(x) \delta_{x}$ is a mapping from $V(G)$ to $\mathbb{N}$ such that $h(x)=f(x)$ and $h\left(x^{\prime}\right)=0$ for $x^{\prime} \neq x$. The mapping $f-f(x) \delta_{x}$ agrees with $f$ on every vertex $x^{\prime} \neq x$ and vanishes on $x$.

We use the following conventions:

- When we define a mapping $f: V(G) \rightarrow \mathbb{N}$, if $f(x)$ is only defined for a subset $U$ of $V(G)$, then it is assumed that $f(x)=0$ for $x \notin U$.

- For a subset $X$ of $V(G), f(X)=\sum_{v \in X} f(v)$.

- For a subset $X$ of $V, G[X]$ is the subgraph of $G$ induced by $X$ and $G-X$ is the subgraph of $G$ induced by $V \backslash X$. 
For a vertex $x$ of $G, N_{G}(x)=\{y:(x, y) \in E(G)\}$ is the set of neighbours of $x$. Let $N_{G}[x]=N_{G}(x) \cup\{x\}$. If there is no confusion, write $N(x)$ for $N_{G}(x)$, and $N[x]$ for $N_{G}[x]$. If $X$ is a subset of $V(G)$, then $N(X)=\cup_{x \in X} N(x)$ and $N[X]=N(X) \cup X$.

Given a graph $G$ and mappings $f, g: V(G) \rightarrow \mathbb{N}$, on-line $(f, g)$-choosability of a graph can be equivalently defined recursively as follows:

- If $g(x)=0$ for all vertices $x \in V(G)$, then $G$ is on-line $(f, g)$-choosable.

- Suppose $g$ is not constantly 0 . Then $G$ is on-line $(f, g)$-choosable if and only if the following hold:

1. $f(x) \geqslant g(x)$ for each vertex $x$.

2. For every non-empty subset $U$ of $V(G)$ with $g \mid U \geqslant 1$, there is an independent set $X$ of $G$ contained in $U$ such that $G$ is on-line $\left(f-\delta_{U}, g-\delta_{X}\right)$-choosable.

Lemmas 3, 4 and 5 below follow easily from the definition.

Lemma 3 If $G$ is on-line $(f, g)$-choosable and $f^{\prime}(x) \geqslant f(x)$ and $g^{\prime}(x) \leqslant g(x)$ for all $x \in V(G)$, then $G$ is on-line $\left(f^{\prime}, g^{\prime}\right)$-choosable.

Lemma 4 If $G$ is on-line $(f, g)$-choosable, then $f \geqslant g$. In case $G$ has no edge, then $G$ is on-line $(f, g)$-choosable if and only if $f \geqslant g$.

Lemma 5 If $G$ is on-line $(f, g)$-choosable and $g(x)=0$, then $G$ is on-line $\left(f-f(x) \delta_{x}, g\right)$ choosable.

Lemma 5 says that if $g(x)=0$, i.e., we do not need to assign any colour to $x$, then any permissible colours assigned to $x$ are useless, and hence can be omitted. In the following, we may assume that $f(x)=0$ whenever $g(x)=0$.

Lemma 6 Suppose $G=(V, E)$ is a graph and $A$ is a subset of $V$. Assume $f, g, f^{\prime}, g^{\prime}$ : $V \rightarrow \mathbb{N}$ are mappings such that for all $x \in A, g^{\prime}(x) \geqslant g(x)$ and

$$
f(x)-g(x)-f^{\prime}(x)+g^{\prime}(x) \geqslant g(N(x) \backslash A) .
$$

If $G-A$ is on-line $(f, g)$-choosable and $G[A]$ is on-line $\left(f^{\prime}, g^{\prime}\right)$-choosable, then $G$ is on-line $(f, g)$-choosable.

Proof. We prove this lemma by induction on $\sum_{x \in V(G)} g(x)$. If $g$ is constant 0 then the conclusion is true. Assume $g$ is not constant 0 . Let $U$ be a non-empty subset of $V(G)$ with $g \mid U \geqslant 1$. We need to find an independent set $X$ contained in $U$ such that $G$ is on-line $\left(f-\delta_{U}, g-\delta_{X}\right)$-choosable.

By assumption, $G-A$ is on-line $(f, g)$-choosable. So there is an independent set $X^{\prime}$ of $G$ contained in $U \backslash A$ such that $G-A$ is on-line $\left(f-\delta_{U}, g-\delta_{X^{\prime}}\right)$-choosable. Let 
$U^{\prime \prime}=(U \cap A) \backslash N\left(X^{\prime}\right)$. As $g^{\prime} \geqslant g$, we have $g^{\prime} \mid U^{\prime \prime} \geqslant 1$. Since $G[A]$ is on-line $\left(f^{\prime}, g^{\prime}\right)$ choosable, there is an independent set $X^{\prime \prime}$ of $G$ contained in $U^{\prime \prime}$ such that $G[A]$ is on-line $\left(f^{\prime}-\delta_{U^{\prime \prime}}, g^{\prime}-\delta_{X^{\prime \prime}}\right)$-choosable. Let $X=X^{\prime} \cup X^{\prime \prime}$. Then $X$ is an independent set of $G$ contained in $U$. We shall show that $G$ is on-line $\left(f-\delta_{U}, g-\delta_{X}\right)$-choosable. By induction hypothesis, it suffices to show that for any $x \in A, g^{\prime}(x)-\delta_{X^{\prime \prime}}(x) \geqslant g(x)-\delta_{X}(x)$ (which is true because $\left.g^{\prime}(x) \geqslant g(x)\right)$ and

$$
\left(f(x)-\delta_{U}(x)\right)-\left(g(x)-\delta_{X}(x)\right)-\left(f^{\prime}(x)-\delta_{U^{\prime \prime}}(x)\right)+\left(g^{\prime}(x)-\delta_{X^{\prime \prime}}(x)\right) \geqslant\left(g-\delta_{X}\right)(N(x)-A) .
$$

Assume $x \in A$. Since $\delta_{X}(x)=\delta_{X^{\prime \prime}}(x)$ and (1) holds, to prove (2), it suffices to show that

$$
\delta_{U}(x)-\delta_{U^{\prime \prime}}(x) \leqslant \delta_{X}(N(x)-A) .
$$

For $x \notin U \cap A-U^{\prime \prime}$, the lefthand side of (3) is 0 , and hence the inequality holds. For $x \in U \cap A-U^{\prime \prime},(3)$ also holds trivially because $x \in N\left(X^{\prime}\right)$ and hence $\delta_{X}(N(x)-A) \geqslant 1$. This completes the proof of Lemma 6 .

In the application of Lemma 6 , usually we have $g^{\prime}=g$. This special case can be stated as follows:

Corollary 7 Suppose $G=(V, E)$ is a graph and $A$ is a subset of $V$. If $G-A$ is on-line $(f, g)$-choosable and $G[A]$ is on-line $(f-\pi, g)$-choosable, where $\pi(x)=g(N(x) \backslash A)$, then $G$ is on-line $(f, g)$-choosable.

Corollary 8 If $x$ is a vertex of $G$ and $f(x) \geqslant \sum_{y \in N_{G}[x]} g(y)$, then $G$ is on-line $(f, g)$ choosable if and only if $G-x$ is on-line $(f, g)$-choosable. In particular, if $f(x) \geqslant(d(x)+$ $1) b$, then $G$ is on-line $(f, b)$-choosable if and only if $G-x$ is on-line $(f, b)$-choosable.

Proof. The "only if" part is obvious. The "if" part follows from Corollary 7 by letting $A=\{x\}$.

Lemma 9 Suppose $G=(V, E)$ is a graph, $A$ is a subset of $V$ and $B \cup B^{\prime}$ is a partition of $N(A) \backslash A$. Let $s=g(A \cup B)$. If $G-A$ is on-line $(f, g)$-choosable, then $G$ is on-line $\left(f^{\prime}, g\right)$-choosable, where $f^{\prime}=f+s \delta_{B^{\prime} \cup A}$.

Proof. We prove the lemma by induction on $f^{\prime}(V)$. Assume $U$ is a subset of $V$ with $g \mid U \geqslant 1$. By the recursive definition of on-line choosability of graphs, it suffices to show that there is an independent set $X$ contained in $U$ such that $G$ is on-line $\left(f^{\prime}-\delta_{U}, g-\delta_{X}\right)$ choosable.

If $U \cap A=\emptyset$, then since $G-A$ is on-line $(f, g)$-choosable, there is an independent set $X$ contained in $U$ such that $G-A$ is on-line $\left(f-\delta_{U}, g-\delta_{X}\right)$-choosable.

Let $s^{\prime}=\left(g-\delta_{X}\right)(A \cup B)$. By induction hypothesis, $G$ is on-line $\left(f^{\prime \prime}, g^{\prime}\right)$-choosable, where

$$
\begin{aligned}
f^{\prime \prime} & =f-\delta_{U}+s^{\prime} \delta_{B^{\prime} \cup A} \\
g^{\prime} & =g-\delta_{X} .
\end{aligned}
$$


Since $s^{\prime} \leqslant s$, by Lemma $3, G$ is on-line $\left(f^{\prime}-\delta_{U}, g-\delta_{X}\right)$-choosable.

Assume $U \cap A \neq \emptyset$. Let $U^{\prime}=U \backslash\left(A \cup B^{\prime}\right)$. Since $G-A$ is on-line $(f, g)$-choosable, there is an independent set $X^{\prime}$ contained in $U^{\prime}$ such that $G-A$ is on-line $\left(f-\delta_{U^{\prime}}, g-\delta_{X^{\prime}}\right)$-choosable. If $X^{\prime} \cap B=\emptyset$, then let $X^{\prime \prime}$ be an independent set of $U \cap A$ and let $X=X^{\prime} \cup X^{\prime \prime}$. Otherwise, let $X=X^{\prime}$. We shall show that in both cases, $G$ is on-line $\left(f^{\prime}-\delta_{U}, g-\delta_{X}\right)$-choosable. By induction hypothesis and by Lemma 3, it suffices to show that for $s^{\prime}=\left(g-\delta_{X}\right)(A \cup B)$,

$$
f-\delta_{U^{\prime}}+s^{\prime} \delta_{B^{\prime} \cup A} \leqslant f^{\prime}-\delta_{U}=f+s \delta_{B^{\prime} \cup A}-\delta_{U}
$$

By the choice of $X$, we know that $X \cap(A \cup B) \neq \emptyset$. Hence $s^{\prime} \leqslant s-1$. Since $\delta_{U}-\delta_{U^{\prime}} \leqslant \delta_{A \cup B^{\prime}}$, we conclude that $\delta_{U}-\delta_{U^{\prime}} \leqslant\left(s-s^{\prime}\right) \delta_{A \cup B^{\prime}}$. Hence inequality (1) holds.

Lemma 10 Suppose $G=(V, E)$ is a graph and $A$ is an independent set such that $f \mid A=$ $g \mid A$. Let $f^{\prime}: V \backslash A \rightarrow \mathbb{Z}$ be defined by $f^{\prime}(u)=f(u)-\sum_{v \in N(u) \cap A} g(v)$. Then $G$ is online $(f, g)$-choosable if and only if $f^{\prime}(u) \geqslant 0$ for every $u \in V \backslash A$ and $G-A$ is on-line $\left(f^{\prime}, g\right)$-choosable.

Proof. By using induction, it suffices to consider the case that $A=\{v\}$ and $g(v) \geqslant 1$. The "if" part follows from Lemma 9 by letting $A=\{v\}, B=\emptyset$ and $B^{\prime}=N(v)$. Now we prove the "only if" part. Assume $G$ is $(f, g)$-choosable. Let $U=N[v]$. By the recursive definition of on-line $(f, g)$-choosability, there is an independent set $X$ contained in $U$ such that $G$ is on-line $\left(f-\delta_{U}, g-\delta_{X}\right)$-choosable. This implies that $\left(f-\delta_{U}\right)(v) \geqslant$ $\left(g-\delta_{X}\right)(v)$. Since $f(v)=g(v)$, we must have $v \in X$, and hence $X=\{v\}$. Therefore $G$ is on-line $\left(f-\delta_{U}, g-\delta_{v}\right)$-choosable. Since $\left(f-\delta_{U}\right)(v)=\left(g-\delta_{X}\right)(v)$, by induction hypothesis, $G-A$ is on-line $\left(f^{\prime \prime}, g\right)$-choosable, where $f^{\prime \prime}: V-A \rightarrow \mathbb{Z}$ is defined as $f^{\prime \prime}(u)=f(u)-\delta_{U}(u)-\sum_{v \in N(u) \cap A}(g(v)-1)=f^{\prime}(u)$. This completes the proof.

\section{Upper bounds for $\mathrm{ch}^{\mathrm{OL}}(G)$ and $\mathrm{ch}_{b}^{\mathrm{OL}}(G)$}

This section gives an upper bound for $\operatorname{ch}^{\mathrm{OL}}(G)$ and an upper bound for $\operatorname{ch}_{b}^{\mathrm{OL}}(G)$ in terms of $\chi(G)$ and $|V(G)|$.

Theorem 11 Suppose $G$ is a graph on $n$ vertices. Then $\operatorname{ch}^{\mathrm{OL}}(G) \leqslant \chi(G) \ln n+1$.

Proof. Assume $\chi(G)=r$ and $A_{1}, A_{2}, \ldots, A_{r}$ are the colour classes. During the game, Police will keep record of a weight function on the vertices of $G$. Initially, $w(v)=1$ for every vertex $v$ of $G$.

Suppose Thief has constructed the set $V_{i}$. Choose an index $j$ such that $w\left(V_{i} \cap A_{j}\right) \geqslant$ $w\left(V_{i} \cap A_{j^{\prime}}\right)$ for all $j^{\prime}$. Let $X_{i}=V_{i} \cap A_{j}$.

Now we modify the weight function. For convenience, we denote the weight function after the modification by $w_{N}$. However, after the modification is finished, we still use $w$ to denote the weight function. Let $w_{N}(v)=0$ for each vertex $v \in X_{i}$, and let $w_{N}(v)=$ 
$\frac{r}{r-1} w(v)$ for each vertex $v \in V_{i} \backslash X_{i}$. There is no change in the weight of other vertices. The increase of the total weight of all the vertices is equal to

$$
w_{N}(V(G))-w(V(G))=\frac{1}{r-1} w\left(V_{i} \backslash X_{i}\right)-w\left(X_{i}\right)
$$

By the choice of $X_{i}, w\left(V_{i} \cap A_{j}\right) \geqslant w\left(V_{i} \cap A_{j^{\prime}}\right)$ for all $j^{\prime}$. Hence $w\left(X_{i}\right) \geqslant \frac{1}{r-1} w\left(V_{i} \backslash X_{i}\right)$. So the increase of the total weight of the vertices is non-positive. Thus if Police applies this strategy, then at any time, the total weight of all the vertices is at most $n$. On the other hand, if a vertex $v$ is contained in $m$ of the $V_{i}$ 's and is not contained in any of the $X_{i}$ 's, then $w(v)=\left(\frac{r}{r-1}\right)^{m}$. Hence $\left(\frac{r}{r-1}\right)^{m} \leqslant n$. Since $\frac{r}{r-1} \geqslant e^{\frac{1}{r}}$, we conclude that $m \leqslant r \ln n$. Thus if a vertex is contained in $r \ln n+1$ of the sets $V_{i}$, then $v$ must be contained in an $X_{i}$.

Theorem 12 Let $G$ be a graph on $n$ vertices and $b$ is a positive integer. Then $\mathrm{ch}_{b}^{\mathrm{OL}}(G) \leqslant$ $\frac{e \chi(G)-1}{e-1}(b-1+\ln n)+b$.

Proof. Similarly as in the proof of Theorem 11, assume $\chi(G)=r$ and $A_{1}, A_{2}, \ldots, A_{r}$ are the colour classes. Police will keep record of a weight function on the vertices of $G$ during the game. Initially, $w(v)=1$ for every vertex $v$ of $G$.

Suppose Thief has constructed the set $V_{i}$. Choose an index $j$ such that $w\left(V_{i} \cap A_{j}\right) \geqslant$ $w\left(V_{i} \cap A_{j^{\prime}}\right)$ for all $j^{\prime}$. Let $X_{i}=V_{i} \cap A_{j}$. We modify the weight function as follows: Let $w_{N}(v)=w(v) / e$ for each vertex $v \in X_{i}$, and let $w_{N}(v)=\frac{e r-1}{e(r-1)} w(v)$ for each vertex $v \in V_{i} \backslash X_{i}$, (similarly, $w_{N}$ is the new weight function, which will be written as $w$ again after the modification is finished). There is no change in the weight of other vertices. The increase of the total weight of all the vertices is equal to

$$
w_{N}(V(G))-w(V(G))=\frac{e-1}{e(r-1)} w\left(V_{i} \backslash X_{i}\right)-\frac{e-1}{e} w\left(X_{i}\right)
$$

By the choice of $X_{i}, w\left(V_{i} \cap A_{j}\right) \geqslant w\left(V_{i} \cap A_{j^{\prime}}\right)$ for all $j^{\prime}$. Hence $\frac{e-1}{e} w\left(X_{i}\right) \geqslant \frac{e-1}{e(r-1)} w\left(V_{i} \backslash X_{i}\right)$. So the increase of the total weight of the vertices is non-positive. Thus if Police applies this strategy, then at any time, the total weight of all the vertices is at most $n$. On the other hand, if a vertex $v$ is contained in $m$ of the $V_{i}$ 's and is coloured with $t$ colours, then $w(v)=\left(\frac{e r-1}{e(r-1)}\right)^{m-t} / e^{t} \leqslant n$. Assume $v$ is coloured with less than $b$ colours, i.e., $t \leqslant b-1$, then $\left(\frac{e r-1}{e(r-1)}\right)^{m-t} \leqslant e^{b-1} n$. Since $\frac{e r-1}{e(r-1)} \geqslant e^{\frac{e-1}{e r-1}}$, we conclude that $m-t \leqslant \frac{e r-1}{e-1}(b-1+\ln n)$. Hence $m \leqslant \frac{e r-1}{e-1}(b-1+\ln n)+b-1$. Thus if a vertex is contained in $\frac{e r-1}{e-1}(b-1+\ln n)+b$ of the sets $V_{i}$, then $v$ must be coloured with $b$ colours.

Suppose a graph $G$ and a $\chi(G)$ colouring of $G$ is given. Let $k=\chi(G) \ln n+1$. Then for any $k$-assignment $L$ of $G$, the proof of Theorem 11 gives a polynomial time algorithm which constructs an $L$-colouring of $G$. We assume that the list of permissible colours for each vertex is a set of positive integers. For $i=1,2, \ldots$, , Let $V_{i}$ be the set of vertices which have colour $i$ in their list. Then we use the method in the proof of Theorem 11 to 
construct colour classes. To accomplish the task, what we need to do is to calculate and update the weight of vertices. The weight of each vertex is updated at most $k$-times. So the running time of the algorithm is $O(k n)=O(n \ln n)$. Let $m=\frac{e \chi(G)-1}{e-1}(b-1+\ln n)+b$. Then for any $m$-assignment $L$ of $G$, the proof of Theorem 12 gives a polynomial time algorithm which constructs an $(L, b)$-colouring of $G$. The running time of the algorithm is also $O(n \ln n)$.

The following theorem is a generalization of Theorem 12. Its proof is similar (modify the weight of vertices in the same way as in the proof of Theorem 12) and omitted.

Theorem 13 Let $g: V(G) \rightarrow \mathbb{N}$ be a given function. Let $p=\sum_{v \in V(G)} e^{g(v)-1}$. If $f(v) \geqslant \frac{e \chi(G)-1}{e-1} \ln p+g(v)$, then $G$ is on-line $(f, g)$-choosable.

\section{On-line 2-choosable graphs}

The class of 2-choosable graphs are characterized in [6]. The core of a connected graph $G$ is the graph obtained from $G$ by successively deleting vertices of degree 1 . The $\theta$-graph $\theta_{a, b, c}$ is the graph consisting of two vertices of degree 3 joined by three internally disjoint paths of respective lengths $a, b, c$.

Theorem 14 [6] A connected graph $G$ is 2-choosable if and only if the kernel of $G$ is one of the following graphs: $K_{1}, C_{2 n}$ or $\theta_{2,2,2 n}$.

In the following, we shall characterize all on-line 2-choosable graphs.

Theorem 15 A connected graph $G$ is on-line 2-choosable if and only if the core of $G$ is one of the following graphs: $K_{1}, C_{2 n}$ or $K_{2,3}$.

Proof. By Corollary 8, a graph $G$ is on-line 2-choosable if and only if the core of $G$ is on-line 2-choosable. Now we show that $K_{1}, C_{2 n}$ and $K_{2,3}$ are on-line 2-choosable. The graph $K_{1}$ is trivial. For $C_{2 n}$, orient the edges of $C_{2 n}$ to form a directed cycle. Then each subset $S$ of $V\left(C_{2 n}\right)$ contains a kernel, i.e., an independent set $X$ such that every vertex in $S-X$ has an out-neighbour in $X$. Whenever Thief chooses a set $V_{i}$, then Police choose a kernel $X_{i}$ in $V_{i}$. Since each vertex has out-degree 1, if a vertex is contained in two $V_{i}$ 's, then it must be chosen by Police. So this is a winning strategy for Police.

The graph $K_{2,3}$ is small and it can be checked easily that it is on-line 2-choosable. We just give the first move of Police: Assume the partite sets are $A=\left\{a_{1}, a_{2}\right\}$ and $B=\left\{b_{1}, b_{2}, b_{3}\right\}$. If $\left|V_{1} \cap B\right| \leqslant\left|V_{1} \cap A\right|$, then let $X_{1}=V_{1} \cap A$. Otherwise, let $X_{1}=V_{1} \cap B$. It can be easily verified that Police will win the game.

Since every on-line 2-choosable graph is 2-choosable, to prove that all the other graphs are not on-line 2-choosable, it suffices to show that $\theta_{2,2,2 n}$ is not on-line 2-choosable for $n \geqslant 2$.

Let $u, v$ be the two vertices of degree 3 , and let the three paths be $\left(u, x_{1}, v\right),\left(u, x_{2}, v\right)$ and $\left(u, y_{1}, y_{2}, \ldots, y_{2 n-1}, v\right)$. 
In the first move, Thief let $V_{1}=\left\{y_{1}, y_{2}\right\}$. If Police chooses $X_{1}=\left\{y_{2}\right\}$, then the following moves of Police are forced:

$$
\begin{gathered}
V_{2}=\left\{y_{1}, u\right\} \Rightarrow X_{2}=\left\{y_{1}\right\} \\
V_{3}=\left\{u, x_{1}, x_{2}\right\} \Rightarrow X_{3}=\{u\} \\
V_{4}=\left\{x_{1}, v\right\} \Rightarrow X_{4}=\left\{x_{1}\right\} .
\end{gathered}
$$

Finally, Thief let $V_{5}=\left\{x_{2}, v\right\}$ and wins the game.

If Police chooses $X_{1}=\left\{y_{1}\right\}$, then the following moves of Police are forced:

$$
\begin{gathered}
V_{2}=\left\{y_{2}, y_{3}\right\} \Rightarrow X_{2}=\left\{y_{2}\right\} \\
V_{3}=\left\{y_{3}, y_{4}\right\} \Rightarrow X_{3}=\left\{y_{3}\right\} \\
\ldots \ldots \\
V_{2 n-1}=\left\{y_{2 n-1}, v\right\} \Rightarrow X_{2 n-1}=\left\{y_{2 n-1}\right\} \\
V_{2 n}=\left\{x_{1}, x_{2}, v\right\} \Rightarrow X_{2 n}=\{v\} \\
V_{2 n+1}=\left\{x_{1}, u\right\} \Rightarrow X_{2 n+1}=\left\{x_{1}\right\} .
\end{gathered}
$$

Finally, Thief let $V_{2 n+2}=\left\{x_{2}, u\right\}$ and wins the game.

\section{On-line 3-choosable complete bipartite graphs}

It is easy to verify that the graphs $\theta_{2,2,2 n}$ are on-line 3 -choosable. So if $\operatorname{ch}(G) \leqslant 2$, then $\operatorname{ch}^{\mathrm{OL}}(G) \leqslant 3$, and the bound is tight.

One naturally asks whether there is an upper bound on the on-line choice number of 3 -choosable graphs. The question seems to be difficult. Unlike for 2-choosable graphs, there is no simple characterization of 3-choosable graphs. Even the characterization of all pairs $p, q$ with 3 -choosable $K_{p, q}(p \leqslant q)$ is not easy. A complete description of all the pairs $(p, q)$ is obtained by combining the works of Mahadev, Roberts and Santhanakrishnan [9] $(p=3, q \leqslant 26$ and $p=4, q \leqslant 18)$, Shende and Tesman [14] $(p=5, q \leqslant 12)$, and O-Donnel [10] $(p=6, q \leqslant 10)$.

In the following, we consider on-line 3-choosability of complete bipartite graphs. We shall frequently use the following observation:

Observation 16 A graph $G$ is on-line $f$-choosable if and only if for any subset $U$ of $V(G)$, there is an independent set $X$ contained in $U$ such that $G-X$ is on-line $\left(f-\delta_{U}\right)$ choosable. 
When we restrict to complete bipartite graphs, there is a more convenient way of recording the information. Given a complete bipartite graph $G=K_{n, m}$ together with a mapping $f: V(G) \rightarrow \mathbb{N}$, we represent it by a pair of integer sequences

$$
\left(f\left(a_{1}\right), f\left(a_{2}\right), \ldots, f\left(a_{n}\right) \| f\left(b_{1}\right), f\left(b_{2}\right), \ldots, f\left(b_{m}\right)\right) .
$$

We call such a pair of integer sequences a configuration. For example $(3,3 \| 2,2,2,2,2)$ represents the bipartite graph $K_{2,5}$ with partite sets $A=\left\{a_{1}, a_{2}\right\}$ and $B=\left\{b_{1}, b_{2}, \ldots, b_{5}\right\}$, and $f\left(a_{i}\right)=3$ for $i=1,2, f\left(b_{i}\right)=2$ for $i=1,2,3,4,5$. Sometimes we write $m \star q$ for $m$ consecutive $q$ 's. For example, the configuration $(3,3 \| 2,2,2,2,2)$ can be written as $(2 \star 3 \| 5 \star 2)$ and $(2,2,3,3 \| 2,2,3,3,3)$ can be written as $(2 \star 2,2 \star 3 \| 2 \star 2,3 \star 3)$.

Given a complete bipartite graph $G=K_{n, m}$ and a mapping $f: V(G) \rightarrow \mathbb{N}$, either Thief has a winning strategy or Police has a winning strategy for the on-line $f$-colouring game. We say $\left(f\left(a_{1}\right) f\left(a_{2}\right) \ldots f\left(a_{n}\right) \| f\left(b_{1}\right) f\left(b_{2}\right) \ldots f\left(b_{m}\right)\right)$ is a Thief winning configuration (or Police winning configuration) if Thief (or Police) has a winning strategy in the corresponding game.

Now given a configuration (for a complete bipartite graph $G=K_{n, m}$ with partite sets $A$ and $B$, together with a mapping $f$ ), we consider one round of the play of the on-line $f$-list colouring game. Thief chooses a subset $U$ of $A \cup B$. Then Police either colours the vertices in $U \cap A$ or he colours the vertices in $U \cap B$ (we may assume that both $U \cap A$ and $U \cap B$ are non-empty, for otherwise the move of Police is trivial). After this round of the play, the game will be played on a new complete bipartite graph $G^{\prime}$ with a new mapping $f^{\prime}$, where $G^{\prime}$ is obtained from $G$ by deleting those coloured vertices, and $f^{\prime}$ is obtained from $f$ by reducing the value of $f(x)$ by 1 for those $x \in U$. Thus given a configuration and a subset $U$ of $A \cup B$, we obtain two new configurations (where $U$ corresponds to a move of Thief, and the two new configurations corresponds to the two different choices of Police). We say these two new configurations are induced by $U$.

For example, take the configuration $(3,3 \| 2,2,2,2,2)$. Let $U=\left\{a_{1}, b_{1}\right\}$. Then the two derived configurations are $(3 \| 1,2,2,2,2)$ and $(2,3 \| 2,2,2,2)$. In the first configuration, Police coloured $a_{1}$, so the partite set $A$ has only one vertex left (we shall re-name this vertex of $A$ by $a_{1}$.) As $b_{1}$ is in $U$, it has consumed 1 colour, and $f^{\prime}\left(b_{1}\right)$ becomes 1 .

With this terminology, by the recursive definition of $(f, g)$-choosability of graph, we have the following observation:

Observation 17 A configuration is a Thief winning configuration if there is a subset $U$ of $A \cup B$ such that the two induced configurations are both Thief winning configurations. Conversely, a configuration is a Police winning configuration if for any subset $U$ of $A \cup B$, one of the two induced configurations is a Police winning configurations.

Using this observation, we can construct more and more complicated Thief winning configurations (or Police winning configurations) from simple ones.

Here is a list of some simple Thief winning configurations and Police winning configurations.

Thief winning: $(2 \star 3 \| 9 \star 2),(2,3 \| 6 \star 2),(1,2,3 \| 6 \star 3),(3 \star 2 \| 3 \star 2),(2,2,3 \| 12 \star 3)$. 
Police winning: $(2 \star 3 \| 8 \star 2),(2,3 \| 5 \star 2),(1,2,3 \| 5 \star 3),(3 \star 2 \| 2 \star 2),(2,2,3 \| 11 \star 3)$.

To see that $(2 \star 3 \| 9 \star 2)$ is Thief winning, we note that $K_{2,9}$ is not $f$-choosable for the given $f$ (and hence not on-line $f$-choosable): let $L\left(a_{1}\right)=\{1,2,3\}$ and $L\left(a_{2}\right)=\{4,5,6\}$, and let $\left\{L\left(b_{i}\right): i=1,2, \ldots, 9\right\}=L\left(a_{1}\right) \times L\left(a_{2}\right)$. It is easy to see that $K_{2,9}$ is not $L$-colourable.

To see that $(2,2,3 \| 12 \star 3)$ is Thief winning, it suffices to note that the two configurations induced by $U=\left\{a_{1}, b_{1}, \ldots, b_{6}\right\}$ are $(2,3 \| 6 \star 2,6 \star 3)$ and $(1,2,3 \| 6 \star 3)$, and both configurations are Thief winning configurations.

Lemma 18 The complete bipartite graph $K_{3, q}$ is on-line 3 -choosable if and only if $q \leqslant 26$.

Proof. If $q \geqslant 27$, then $K_{3, q}$ is not 3 -choosable and hence not on-line 3-choosable. It remains to show that $G=K_{3,26}$ is on-line 3 -choosable. I.e., $(3 \star 3 \| 26 \star 3)$ is a Police winning configuration. To prove this, we need to show that for each $U \subseteq V(G)$, at least one of the configuration induced by $U$ is a Police winning configuration.

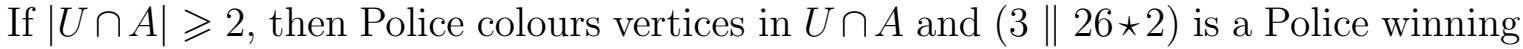
configuration (by using Lemma 6).

Assume that $U \cap A=\left\{a_{3}\right\}$. If $|U \cap B| \leqslant 8$, then Police colours the vertex $a_{3}$ and $(3,3 \| 8 \star 2,18 \star 3)$ is a Police winning configuration. (By using Lemma 6 , we may delete those vertices $x$ in $B$ with $f(x)=3$, and hence it suffices to show that $(3,3 \| 8 \star 2)$ is a Police winning configuration, which is easy.)

Assume that $|U \cap B| \geqslant 9$. Then Police colours vertices in $U \cap B$, and we claim that $(2,3,3 \| 17 \star 3)$ is a Police winning configuration. The proof of this claim is routine check, but there are some cases and we omit the details.

It follows from Lemma 18 that a complete graph $K_{3, q}$ is on-line 3-choosable if and only if it is 3-choosable. The next result shows that there are complete graphs of the form $K_{6, q}$ which are 3 -choosable but not on-line 3 -choosable, namely $K_{6,9}$ and $K_{6,10}$.

Lemma 19 If $q \geqslant 9$, then $K_{6, q}$ is not on-line 3 -choosable.

Proof. It suffices to prove that $K_{6,9}$ is not on-line 3-choosable. Figure 1 below gives a winning strategy for Thief.

The root is the configuration $(6 \star 3 \| 9 \star 3)$. We want to prove that this is a Thief winning configuration. For this purpose we choose a set $U$, which is indicated in the figure by upper bars, i.e., $U=\left\{a_{1}, a_{2}, a_{3}, b_{1}, b_{2}, b_{3}, b_{4}\right\}$. Now $U$ induces two configurations, which are the two sons of the root. We need to show that each of these two sons is a Thief winning configuration. We repeat this process, until we reach the leaves, which are obviously Thief winning configurations. For example, the left most leaf is labeled by (22233\|222). This graph contains the complete bipartite graph $K_{3,3}$ as a subgraph, with each vertex having 2 permissible colours. But $K_{3,3}$ is not 2-choosable. So this is a winning configuration for Thief. The rightmost leaf is labeled by $(22|| 222233333)$. This is a winning configuration for Thief as $K_{2,4}$ is not 2-choosable. The other leaves are obviously winning configurations for Thief. 


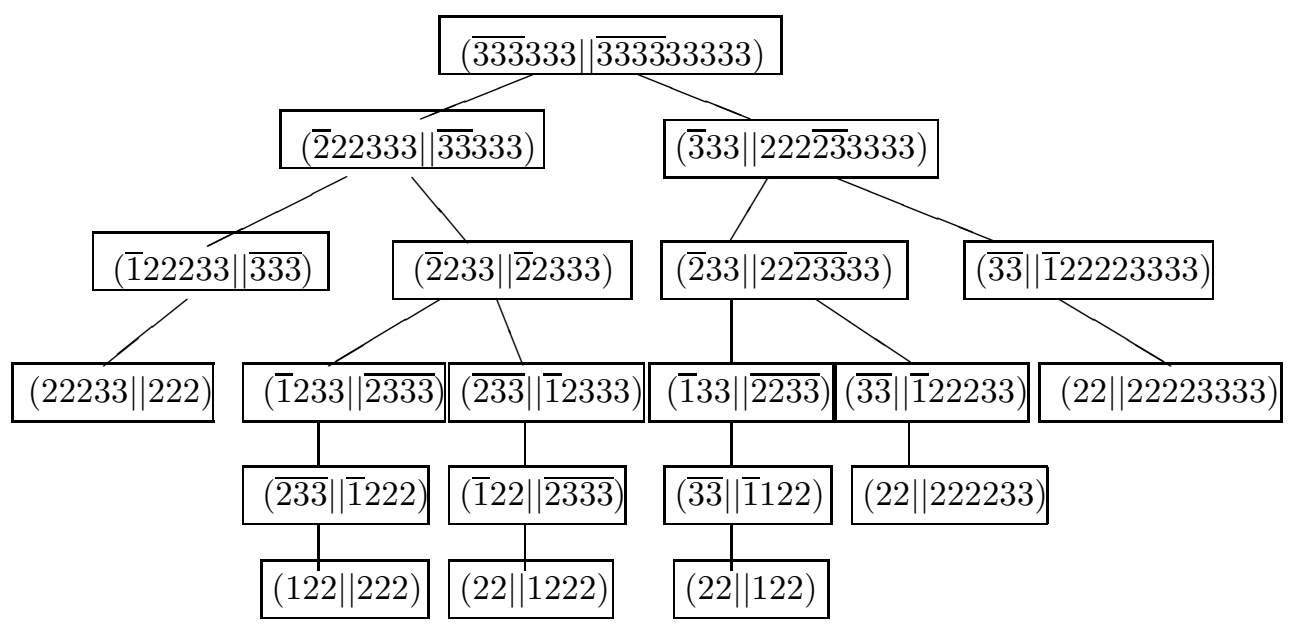

Figure 1: A winning strategy for Thief

\section{Some open questions}

The most natural question concerning the on-line choice number is the relation between the choice number and the on-line choice number of a graph. It follows from the definition that for any graph $G, \operatorname{ch}(G) \leqslant \operatorname{ch}^{\mathrm{OL}}(G)$. We have seen example graphs $G$ for which $\operatorname{ch}(G)<\operatorname{ch}^{\mathrm{OL}}(G)$.

Question 20 Can the difference $\mathrm{ch}^{\mathrm{OL}}(G)-\operatorname{ch}(G)$ be arbitrarily large? If yes, then can the ratio $\frac{\mathrm{ch}^{\mathrm{OL}}(G)}{\operatorname{ch}(G)}$ be arbitrarily large?

It was shown by Alon [2] that if a graph $G$ has average degree at least $4\left(\begin{array}{c}s^{4} \\ s\end{array}\right) \log _{2}\left(2\left(\begin{array}{c}s^{4} \\ s\end{array}\right)\right)$, then $\operatorname{ch}(G)>s$. It follows that if $\operatorname{ch}(G) \leqslant s$, then $G$ has colouring number at most $4\left(\begin{array}{c}s^{4} \\ s\end{array}\right) \log _{2}\left(2\left(\begin{array}{c}s^{4} \\ s\end{array}\right)\right)$ and by Lemma $6, \operatorname{ch}^{\mathrm{OL}}(G) \leqslant 4\left(\begin{array}{c}s^{4} \\ s\end{array}\right) \log _{2}\left(2\left(\begin{array}{c}s^{4} \\ s\end{array}\right)\right)$. So there is an upper bound of $\mathrm{ch}^{\mathrm{OL}}(G)$ in terms of $c h(G)$. However, this upper bound is exponential. If the answer to the second question in Question 20 is negative, then $\operatorname{ch}^{\mathrm{OL}}(G)$ is bounded by a linear function of $c h(G)$. As a weaker question, we may ask:

Question 21 Is $\mathrm{ch}^{\mathrm{OL}}(G)$ bounded by a polynomial function of $\operatorname{ch}(G)$ ?

We have shown that if $\operatorname{ch}(G) \leqslant 2$, then $\operatorname{ch}^{\mathrm{OL}}(G) \leqslant 3$.

Question 22 What is the maximum value of $\mathrm{ch}^{\mathrm{OL}}(G)$ for 3-choosable graphs?

It is proved by Alon and Krivelevich [3] that random bipartite graphs $G_{n, n, p}$ (i.e., each partite set has $n$ vertices and each edge added to the graph with probability $p$ ) almost surely has choice number $(1+o(1)) \log _{2}(n p)$. Alon and Krivelevich [3] also conjectured that for any bipartite graph $G$ with maximum degree $d, c h(G)=O(\ln d)$. 
Question 23 Is it true that $\mathrm{ch}^{\mathrm{OL}}\left(G_{n, n, p}\right)$ is almost surely at most $(1+o(1)) \log _{2}(n p)$ ? Is it true that for any bipartite graph $G$ with maximum degree $d, \mathrm{ch}^{\mathrm{OL}}(G)=O(\ln d)$ ?

It was conjectured by Erdös, Rubin and Taylor [6] that if $G$ is $k$-choosable, then for any positive integer $b, G$ is $(k b, b)$-choosable. The conjecture is still open. The same question is also interesting for on-line list colouring.

Question 24 Is it true that for any graph $G$ and for any positive integer $b, \operatorname{ch}_{b}^{\mathrm{OL}}(G) \leqslant$ $b \mathrm{ch}^{\mathrm{OL}}(G)$ ?

The choice ratio of a graph is defined as $\inf \left\{\frac{c h_{b}(G)}{b}: b \in \mathbb{N}\right\}$. It is proved in [4] that the choice ratio of a graph $G$ is equal to its fractional chromatic number. We may define the on-line choice ratio of $G$ as $\inf \left\{\frac{\operatorname{ch}_{b}^{\mathrm{OL}}(G)}{b}: b \in \mathbb{N}\right\}$.

Question 25 Is it true that the on-line choice ratio of any graph equals its fractional chromatic number?

A famous conjecture of Erdös-Lovasz-Farber [5] says that if $G$ is the edge-disjoint union of $n$ complete graphs of order $n$ each, then $\chi(G)=n$. Alon (see Page 13 of [16]) further conjectured that such a graph has choice number $n$.

Question 26 If $G$ is the edge-disjoint union of $n$ complete graphs of order $n$ each, is it true that $\operatorname{ch}^{\mathrm{OL}}(G)=n$ ?

Question 26 is much stronger than the original Erdös-Lovász-Farber Conjecture. So one might first try to find a counterexample. Basically, any question we ask for the choice number of graphs is very much likely to be meaningful for the on-line choice number of graphs. Sometimes we obtain a stronger statement, such as Question 26. Sometimes the answer to question for the choice number is independent to the answer to the corresponding question for on-line choice number. It is a well-known open question whether $\operatorname{ch}\left(G_{1} \cup G_{2}\right) \leqslant \operatorname{ch}\left(G_{1}\right) \operatorname{ch}\left(G_{2}\right)$, where $G_{1} \cup G_{2}$ is the edge-disjoint union of two graphs $G_{1}$ and $G_{2}$ on the same vertex-set.

Question 27 Suppose $G_{1}$ and $G_{2}$ are graphs on the same vertex-set. Is it true that $\operatorname{ch}^{\mathrm{OL}}\left(G_{1} \cup G_{2}\right) \leqslant \operatorname{ch}^{\mathrm{OL}}\left(G_{1}\right) \operatorname{ch}^{\mathrm{OL}}\left(G_{2}\right)$ ? As a special case, if $G$ is a bipartite graph and $G^{\prime}$ is obtained from $G$ by substituting each vertex with two nonadjacent vertices, is it true that $\operatorname{ch}^{\mathrm{OL}}\left(G^{\prime}\right) \leqslant\left(\operatorname{ch}^{\mathrm{OL}}(G)\right)^{2}$ ?

A positive answer to Question 24 implies a positive answer to Question 27. To prove this, let $a=\operatorname{ch}^{\mathrm{OL}}\left(G_{1}\right)$ and $b=\mathrm{ch}^{\mathrm{OL}}\left(G_{2}\right)$. A winning strategy for Police in the on-line $a b$-list colouring game for $G_{1} \cup G_{2}$ is as follows: When Thief constructs a set $V_{i}$, Police uses his strategy for the on-line list $b$-colouring game on $G_{1}$, obtaining a set $X_{i}$ which is an independent set of $G_{1}$. Then by viewing $X_{i}$ as a subset of $V\left(G_{2}\right)$, Polices uses his winning strategy on the on-line list colouring game on $G_{2}$, to an independent set $Y_{i}$ of $G_{2}$ contained in $X_{i}$. Then $Y_{i}$ is an independent set of $G=G_{1} \cup G_{2}$. If each vertex of $G$ 
is contained in $a b$ of the $V_{i}$ 's, then each vertex is contained in $b$ of the $X_{i}$ 's, and hence contained in at least one of the $Y_{i}$ 's. So this is indeed a winning strategy for Police.

It is proved by Alon [1] that for every fixed edge probability $p, \operatorname{ch}\left(G_{n, p}\right)=(1+o(1))$. $\chi\left(G_{n, p}\right)$ with probability $1-o(1)$ as $n \rightarrow \infty$.

Question 28 Is it true that for every fixed edge probability $p$,

$$
\operatorname{ch}^{\mathrm{OL}}\left(G_{n, p}\right)=(1+o(1)) \cdot \chi\left(G_{n, p}\right)
$$

with probability $1-o(1)$ as $n \rightarrow \infty$ ?

It is proved by Bollobás that

$$
\chi\left(G_{n, p}\right)=\left(\frac{1}{2}+o(1)\right)\left(\log \frac{1}{1-p}\right) \frac{n}{\log n} .
$$

In the Thief-Police game, if Police cannot put all the thieves into prison, a natural question is how many thieves can be put into prison. This motivates the following question:

Question 29 Suppose $G$ is on-line $k$-choosable, and a sequence of subsets $V_{1}, V_{2}, \ldots, V_{m}$ is given sequentially. If each vertex is contained in $k^{\prime}<k$ of the $V_{i}$ 's, is it possible that Police can choose the independent sets $X_{i}$ 's sequentially so that at least $\frac{k^{\prime}}{k}|V(G)|$ vertices are contained in the union of the $X_{i}$ 's? In other words, in the Thief-Police game, can Police guarantee that at least $\frac{k^{\prime}}{k}$ of the thieves are put into prison?

If $k$ is a multiple of $k^{\prime}$, then the non-game version of the question has a positive answer: If $k=a k^{\prime}$ for some positive integer $a, G$ is $k$-choosable and $L$ is a $k^{\prime}$-assignment, then there is an induced subgraph $H$ of $G$ with at least $\frac{k^{\prime}}{k}|V(G)|$ vertices which is $L$-colourable.

Indeed, one can obtain a $k$-assignment $L^{\prime}$ of $G$ by replacing each colour $i$ in $L(v)$ with a colours $(i, 1),(i, 2), \ldots,(i, a)$. Let $f$ be an $L^{\prime}$-colouring of $G$. For $j=1,2, \ldots, a$, let $H_{j}$ be the subgraph of $G$ induced by vertices with colour $(i, j)$ for some $i$. Then there is an index $j$ for which $H_{j}$ contains at least $|V(G)| / a=\frac{k^{\prime}}{k}|V(G)|$ vertices, and $H_{j}$ is $L$-colourable.

If $k$ is not a multiple of $k^{\prime}$, then the non-game version of Question 29 is open. In particular, Albertson [15] asked the following question: Suppose $G$ is 3-choosable, and $L$ is a 2-assignment of $G$. Is it true that there is an induced subgraph $H$ with $\frac{2}{3}|V(G)|$ vertices which is $L$-colourable?

For general functions $f, g$, the $(f, g)$-choosability and the on-line $(f, g)$-chooosability of graphs seem difficult even for graphs of very simple structure. The following question is open.

Question 30 Suppose $T$ is a tree. Characterize those pairs of mappings $f, g$ such that $T$ is $(f, g)$-choosable. Characterize those pairs of mappings $f, g$ such that $T$ is on-line $(f, g)$-choosable. 


\section{Acknowledgement}

The author would like thank Claude Tardif for valuable discussions.

\section{References}

[1] N. Alon. Choice numbers of graphs: a probabilistic approach. Combin. Probab. Comput., 1(2):107-114, 1992.

[2] N. Alon. Restricted colorings of graphs. In Surveys in Combinatorics, 1993 (Keele), volume 187 of London Math. Soc. Lecture Note Ser., pages 1-33. Cambridge Univ. Press, Cambridge, 1993.

[3] N. Alon and M. Krivelevich. The choice number of random bipartite graphs. Ann. Comb., 2(4):291-297, 1998.

[4] N. Alon, Zs. Tuza, and M. Voigt. Choosability and fractional chromatic numbers. Discrete Math., 165/166:31-38, 1997. Graphs and combinatorics (Marseille, 1995).

[5] P. Erdős. On the combinatorial problems which I would most like to see solved. Combinatorica, 1(1):25-42, 1981.

[6] P. Erdős, A. L. Rubin, and H. Taylor. Choosability in graphs. Congress. Numer., 26:125-157, 1980.

[7] S. Gutner and M. Tarsi. Some results on ( $a: b)$-choosability. Discrete Math., 309:2260-2270, 2009.

[8] J. Hladky, D. Král, and U. Schauz. Algebraic proof of Brooks' theorem, manuscript, 2009.

[9] N. V. R. Mahadev, F. S. Roberts, and P. Santhanakrishnan. 3-choosable complete bipartite graphs. Technical Report 49-91, Rutgers University, New Brunswick, NJ, 1991.

[10] P. O-Donnel. (1995), communicated by Z. Tuza, 2009.

[11] U. Schauz. Mr. Paint and Mrs. Correct. Electron. J. Combin., 16(1):\# R77, 2009.

[12] U. Schauz. Flexible color lists in Alon and Tarsi's theorem, and time scheduling with unreliable participants. Electron. J. Combin., submitted.

[13] U. Schauz. Paintability version of the combinatorial nullstellensatz, and list colorings of $k$-partite $k$-uniform hypergraphs. Electron. J. Combin., to appear.

[14] A.M. Shende and B. Tesman. 3-choosability of $k_{5, q}$. CoNum 111(1995), 193-221.

[15] C. Tardif. personal communication.

[16] Z. Tuza. Graph colorings with local constraints - a survey. Discuss. Math. Graph Theory, 17(2):161-228, 1997.

[17] V. G. Vizing. Coloring the vertices of a graph in prescribed colors. Diskret. Analiz, (29 Metody Diskret. Anal. v Teorii Kodov i Shem):3-10, 101, 1976. 